\title{
Relationship between insulin-sensitive obesity and retinal microvascular abnormalities
}

\author{
Wei Lin ${ }^{1 \#}$, Huiying Rao ${ }^{2 \#}$, Huibin Huang ${ }^{1}$, Jin Yao ${ }^{1}$, Jixing Liang ${ }^{1}$, Liantao ${ }^{1}{ }^{1}$, Junping Wen ${ }^{1 \wedge}$, \\ Gang Chen ${ }^{1 \wedge}$
}

${ }^{1}$ Department of Endocrinology, Fujian Provincial Hospital, Shengli Clinical Medical College of Fujian Medical University, Fuzhou, China; ${ }^{2}$ Department of Ophthalmology, Fujian Provincial Hospital, Shengli Clinical Medical College of Fujian Medical University, Fuzhou, China

Contributions: (I) Conception and design: G Chen, W Lin; (II) Administrative support: G Chen, J Wen; (III) Provision of study materials or patients: H Huang, J Yao; (IV) Collection and assembly of data: W Lin, J Liang, L Li; (V) Data analysis and interpretation: W Lin, H Rao, J Wen; (VI) Manuscript writing: All authors; (VII) Final approval of manuscript: All authors.

\#These authors contributed equally to this work.

Correspondence to: Gang Chen. Department of Endocrinology, Fujian Provincial Hospital, Shengli Clinical Medical College of Fujian Medical University, Fuzhou 350001, China. Email: chengangfj@163.com.

Backgroundk Obesity and insulin resistance (IR) are risk factors for microvascular disease (MVD). Whether insulin-sensitive obese individuals are at higher risk for MVD is still debated. We aimed to investigate whether insulin-sensitive obesity is associated with retinal microvascular abnormalities.

Methods: This cross-sectional study recruited a total of 8,313 participants (3,604 males and 4,709 females) aged 21 years or older from 13 villages in rural area and 8 communities in urban area in Fujian province in China between 2011 to 2012. Participants were categorized by insulin-sensitive/-resistant and general/ abdominal obese status. IR was defined as homeostasis model assessment of IR $>1.99$ (75\% percentile). Direct ophthalmoscopic examination was used to diagnose MVD, which was defined as the presence of retinal microvascular abnormalities.

Results: Among the older subjects (aged 65-79 years), those who were obese had a markedly higher risk for MVD, both in insulin-sensitive [odds ratio (OR): 2.259, 95\% confidence interval (CI): 1.041-4.904, $\mathrm{P}=0.039$ ] and insulin-resistant (OR: 2.356, 95\% CI: 1.064-5.218, $\mathrm{P}=0.035$ ) individuals. Additionally, insulinsensitive/-resistant abdominal obesity in middle-aged people showed an increased risk for prevalent MVD in women (OR: 2.061, 3.322; 95\% CI: 1.004-4.233, 1.645-6.709; P values: 0.049, <0.001).

Conclusions: In this study, obesity was closely related to an elevated higher risk of MVD, regardless of IR, especially in older people and abdominally obese middle-aged women. Obesity may be similar to diabetes mellitus, with chronic complications that not only cause cardiovascular diseases but also MVD. Direct ophthalmoscopy may be a noninvasive and meaningful method of early screening for obesity-related MVD. Insulin-sensitive obesity, like impaired glucose tolerance, is a pre-existing state of insulin resistant obesity.

Keywords: Insulin-sensitive obesity; obesity; retinal microvascular abnormalities

Submitted Feb 19, 2020. Accepted for publication Aug 18, 2020.

doi: $10.21037 /$ apm-20-447

View this article at: http://dx.doi.org/10.21037/apm-20-447

^ ORCID: Wei Lin: ORCID: 0000-0002-0455-8752; Junping Wen: ORCID: 0000-0002-5650-5281; Gang Chen: ORCID: 0000-00028105-2384. 


\section{Introduction}

The increasing prevalence of overweight and obesity in many countries (1) has been described as a global pandemic (2). In particular, obesity rates are rising in many developing Asian countries, including China. This has resulted in a shift in the focus of health policy from under-nutrition to obesity and obesity-related diseases (3) because of the increased risks for diabetes mellitus, coronary artery disease and some types of cancer (4).

In recent years, obesity has been divided into two types: insulin-resistant obesity and insulin-sensitive obesity (5). Several studies (6-10) have revealed obesity-related cardiovascular complications in insulin-sensitive obesity. However, the effect of overweight/obesity on microvascular disease (MVD), especially the relationship between insulinsensitive obesity and MVD, is unclear. Whether insulinsensitive obesity is similar to impaired glucose tolerance (IGT), which has chronic complications including not only cardiovascular diseases but also microvascular disorders, is of interest.

As far as we know, the human retinal microcirculation can be measured non-invasively and provides a window to the microvasculature. Recent population-based studies (11-13) have documented subtle retinal vascular changes that occur in metabolic disorders such as diabetes and metabolic syndrome, providing new understanding of the microvascular involvement in these disorders. It has now become evident that these retinal vascular changes might be markers of early preclinical stages of metabolic disorders and may predict their clinical onset. Therefore, in our study, MVD was defined as the presence of retinal microvascular abnormalities.

Given that Asians have a unique phenotype characterized by relatively high abdominal obesity and tend to have a high prevalence of microvascular risk factors even at low body mass index (BMI), we hypothesized that both general and abdominal obesity are risk factors for retinal microvascular abnormalities independent of insulin resistance (IR), and that insulin-sensitive obesity could also be responsible for high retinal microvascular abnormalities. In this study, we investigated the association between insulin-sensitive obesity and MVD in a Chinese population.

We present the following article in accordance with the STROBE reporting checklist (available at http://dx.doi. org/10.21037/apm-20-447).

\section{Methods}

\section{Study cobort}

This cross-sectional study was performed between June, 2011, and January, 2012, and involved participants from 13 villages in rural area and 8 communities in urban area that were randomly selected using a clustered sampling technique with probabilities proportionate to the size of the population in each cluster in Fujian, southeast China. A total of 10,906 Chinese individuals who did not have cognitive dysfunction and were not pregnant (aged between 20 and 79 years old) responded to the survey. The following exclusion criteria were applied: (I) incomplete data for IR and obesity $(\mathrm{n}=256)$; (II) receiving antidiabetic treatment or fasting plasma glucose $(\mathrm{FPG}) \geq 7.0 \mathrm{mmol} / \mathrm{L}$, or 2 -h post-prandial glucose $(2 \mathrm{hPPG}) \geq 11.1 \mathrm{mmol} / \mathrm{L}$ ( $\mathrm{n}=656)$; and (III) refused to undergo retinal examination $(n=1,681)$. A total of 8,313 participants were included in the final analysis (Figure 1). Written informed consent was given by every participant and the study was approved by the Ethics Committee of Fujian Provincial Hospital. The number/ID of the approval is (K2009-12-020). The study was conducted in accordance with the Declaration of Helsinki (as revised in 2013). All investigators, who were unaware of the aims of the study and blinded to the characteristics of the subjects, received special training before the investigation.

\section{Data collection}

All participants completed a standard self-reported questionnaire with 19 questions addressing age, sex, personal and family medical history, age at menarche, age at menopause, parity, smoking and drinking habits, and physical activity. Age at menarche was defined as age at first menstrual period. Postmenopausal was defined as lack of menstrual bleeding for at least 12 months or a history of hysterectomy or oophorectomy, and age of menopause was defined as age at last menstruation.

Weight, height, and waist and hip circumferences were measured to the nearest $0.1 \mathrm{~kg}$ and $0.1 \mathrm{~cm}$, respectively, by experienced nurses with the subjects lightly clothed and without shoes. BMI was calculated as weight in kilograms divided by height in meters squared $\left(\mathrm{kg} / \mathrm{m}^{2}\right)$. Waist circumference was measured at the middle point between the costal margin and iliac crests. The waist-to-hip ratio 


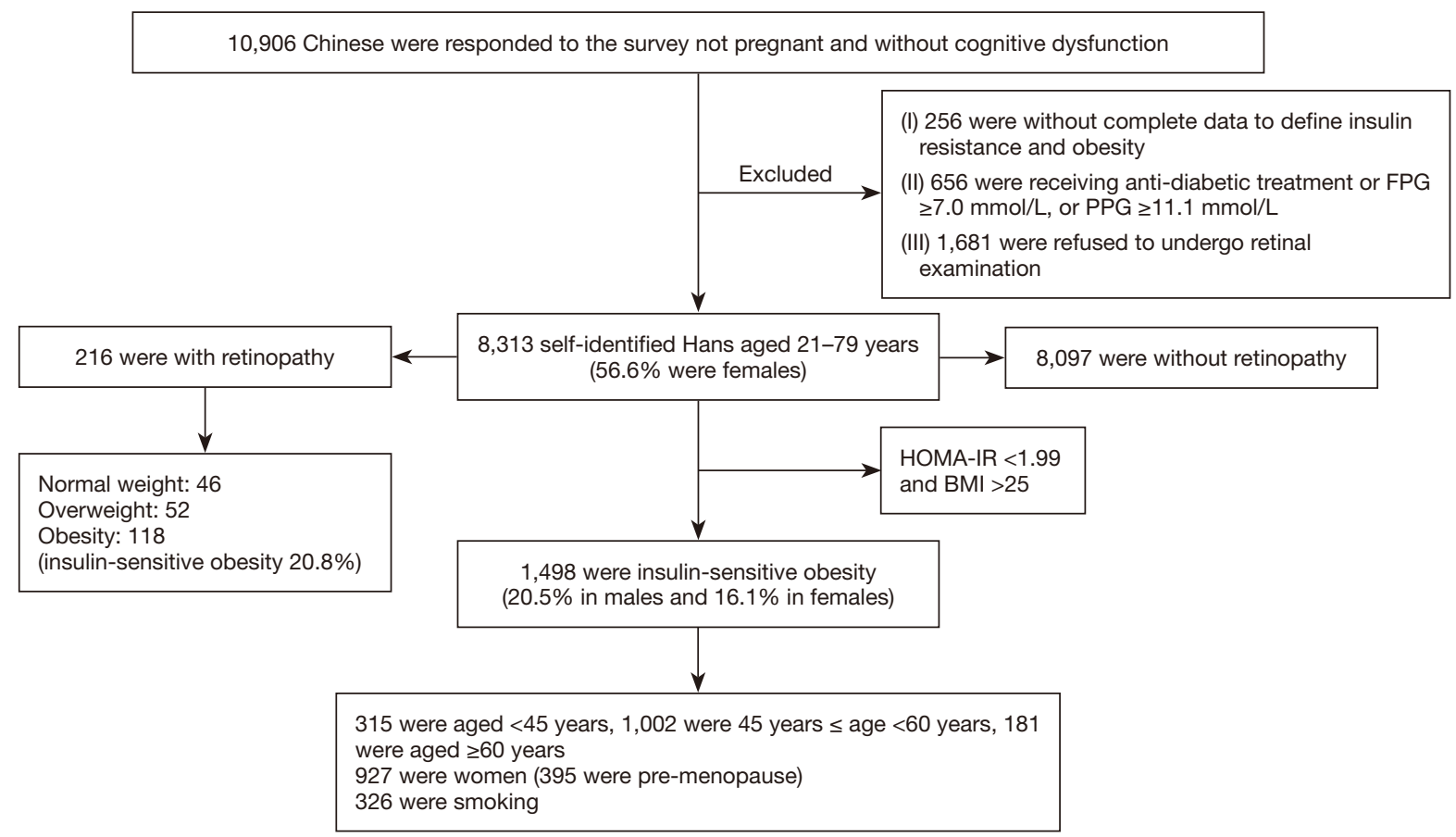

Figure 1 Study flowchart. FPG, fasting plasma glucose; PPG, postprandial blood glucose; HOMA-IR, homeostasis model assessmentestimated insulin resistance; BMI, body mass index.

was the waist circumference divided by hip circumference. Systolic and diastolic blood pressures (SBP and DBP) were both measured three times using a standard OMRON autoelectronic sphygmomanometer and the mean of the three readings was used for analysis.

Blood samples were collected after overnight fasting for at least $10 \mathrm{~h}$. Standard laboratory tests determined FPG, 2hPPG, hemoglobin A1c, fasting serum insulin (FIN), serum creatinine ( $\mathrm{SCr}$ ), total cholesterol (CHOL), total triglycerides (TG), low-density lipoprotein cholesterol (LDL-C), and high-density lipoprotein cholesterol (HDL-C). The index of homeostasis model assessment of IR $($ HOMA-IR) was calculated as: FPG $(\mathrm{mmol} / \mathrm{L}) \times \mathrm{FIN}$ $(\mu \mathrm{U} / \mathrm{mL}) / 22.5$.

Direct ophthalmoscopic examination of both eyes after 5 minutes of adaptation to the dark was conducted independently by two qualified ophthalmologists. A research ophthalmologist undertook a quality assurance check of $10 \%$ of the diagnoses. As a result, no instances of discordance in diagnosis were identified. MVD was defined as present if any of the following lesions were definite in any of the fundus quadrants: microaneurysms, retinal hemorrhages (blot or flame-shaped), soft exudates (cottonwool spots), hard exudates, macular edema, intraretinal microvascular abnormalities, venous beading, new vessels at the disc or elsewhere, vitreous hemorrhage, disc swelling, laser photocoagulation scars, arteriovenous nicking, or focal arteriolar narrowing.

General overweight was defined as BMI $23.0-25.0 \mathrm{~kg} / \mathrm{m}^{2}$ and obesity was defined as BMI $\geq 25.0 \mathrm{~kg} / \mathrm{m}^{2}$, according to Asia-Pacific definitions. Abdominal obesity was defined using the modified National Cholesterol Education Program-Adult Treatment Panel III as waist circumference $\geq 90 \mathrm{~cm}$ for men and $\geq 80 \mathrm{~cm}$ for women. IR was defined as HOMA-IR $>1.99$ (75\% percentile). Diabetes mellitus was defined as $\mathrm{FPG} \geq 7.0 \mathrm{mmol} / \mathrm{L}$ or $2 \mathrm{hPPG} \geq 11.1 \mathrm{mmol} / \mathrm{L}$, a reported previous diagnosis of diabetes or use of hypoglycemic medications. We defined abnormal glomerular filtration rate as $<60 \mathrm{~mL} / \mathrm{min} / 1.73 \mathrm{~m}^{2}$.

\section{Statistical analysis}

EpiData software (The EpiData Association, Odense, Denmark) was used to establish the database. All data were double entered and then compared and corrected for errors. Because sex, age, smoking, and menstrual status have a significant effect on MVD, to better identify the relationships between insulin-sensitive obesity and retinal 
Table 1 Descriptive data for insulin-resistant/-sensitive general obese phenotypes

\begin{tabular}{|c|c|c|c|c|c|c|}
\hline Variables & \multicolumn{2}{|c|}{ Normal weight } & \multicolumn{2}{|c|}{ Overweight } & \multicolumn{2}{|c|}{ Obese } \\
\hline Number & 2,956 & 324 & 1,625 & 485 & 1,498 & 1,425 \\
\hline Age, years & 51 (45 to 59 ) & 53 (46 to 60 ) & 51 (46 to 59 ) & 53 (46 to 61 ) & 53 (46 to 60 ) & 53 (46 to 61$)$ \\
\hline BMI & $\begin{array}{c}21.25 \\
\text { (20.05 to } 22.11)\end{array}$ & $\begin{array}{c}22.04 \\
\text { (21.21 to } 22.54)\end{array}$ & $\begin{array}{c}23.93 \\
\text { (23.46 to } 24.42)\end{array}$ & $\begin{array}{c}24.19 \\
\text { (23.63 to } 24.62)\end{array}$ & $\begin{array}{c}26.50 \\
\text { (25.64 to } 27.75)\end{array}$ & $\begin{array}{c}27.47 \\
\text { (26.20 to } 29.34)\end{array}$ \\
\hline FPG (mmol/L) & 5.26 (4.94 to 5.62$)$ & 5.70 (5.32 to 6.34$)$ & 5.34 (5.01 to 5.72$)$ & 5.69 (5.35 to 6.14$)$ & 5.41 (5.07 to 5.77$)$ & $5.82(5.42$ to 6.38$)$ \\
\hline 2hPPG (mmol/L) & 6.15 (5.20 to 7.32$)$ & 7.00 (5.98 to 8.33$)$ & 6.43 (5.33 to 7.59$)$ & 7.36 (6.29 to 9.07$)$ & 6.56 (5.47 to 7.77$)$ & 7.65 (6.28 to 9.88$)$ \\
\hline $\mathrm{SBP}(\mathrm{mmHg})$ & 125 (115 to 138$)$ & 132 (119 to 147$)$ & 130 (120 to 143$)$ & 133 (121 to 147$)$ & 135 (123 to 147$)$ & 141 (128 to 153$)$ \\
\hline HDL-C (mmol/L) & 1.48 (1.27 to 1.72$)$ & 1.33 (1.13 to 1.52$)$ & 1.34 (1.17 to 1.58$)$ & 1.29 (1.12 to 1.48$)$ & 1.32 (1.13 to 1.52$)$ & 1.25 (1.08 to 1.43$)$ \\
\hline TG (mmol/L) & 1.04 (0.78 to 1.45$)$ & 1.48 (1.03 to 2.26$)$ & $1.26(0.94$ to 1.79$)$ & 1.66 (1.18 to 2.43$)$ & 1.45 (1.06 to 2.08$)$ & $1.81(1.30$ to 2.63$)$ \\
\hline MVD & $37(17.1)$ & $9(4.2)$ & $34(15.7)$ & $18(8.3)$ & $45(20.8)$ & 73 (33.8) \\
\hline
\end{tabular}

Data are presented as median (interquartile range) for continuous variables, or numbers (percentages) for categorical parameters. BMI, body mass index; HOMA-IR, homeostasis model assessment-estimated insulin resistance; FPG, fasting plasma glucose; 2hPPG, 2-hour postprandial blood glucose; SBP, systolic blood pressure; DBP, diastolic blood pressure; CHOL, total cholesterol; LDL-C, low-density lipoprotein cholesterol; HDL-C, high-density lipoprotein cholesterol; TG, triglyceride.

microvascular abnormalities, we conducted an analysis of the total population stratified by age $(<45 / \geq 45$ years and $<65 / \geq 65$ years), and the insulin-sensitive subjects were divided into male and female subgroups. The subgroup of men was stratified by age and smoking status (i.e., $<65 / \geq 65$ years and smoking/non-smoking), and the subgroup of women was stratified by menopausal status (i.e., premenopausal/postmenopausal $<10$ years/postmenopausal $\geq 10$ years). Continuous variables are shown as median with interquartile ranges (IQR, 25th-75th percentile) due to the non-normal distribution, and categorical variables are expressed as counts and percentages.

Multivariate logistic regression analysis was used to test the determinants of retinal microvascular abnormalities and which fundus examination was used as the dichotomous variable. First, the crude associations were evaluated (unadjusted) and then all analyses included adjustment for age (Model 1). Multiple models were built with inclusions of age, FPG, 2hPPG, SBP, DBP, HDL-C, LDL-C, CHOL, TG, smoking status, and alcohol (Model 2). If the subgroup was stratified by age, Model 3 was adjusted for FPG,
2hPPG, SBP, DBP, HDL-C, LDL-C, CHOL, TC, smoking status, alcohol, BMI, and HOMA-IR. If the subgroup was stratified by smoking status, Model 4 was adjusted for age, FPG, 2hPPG, SBP, DBP, HDL-C, LDL-C, CHOL, TG, and alcohol.

All data analyses were performed with SPSS 19.0 statistical software package (SPSS, Chicago, IL, USA). All $\mathrm{P}$ values were based on two-sided tests, with statistical significance defined as $\mathrm{P}<0.05$.

\section{Results}

A total of 8,313 participants [median age, 52 years (IQR, 46-60 years), range, $21-79$ years], comprising 3,604 (43.4\%) men and 4,709 (56.6\%) women, were included in the analysis. Among them, 1,498 subjects had insulin-sensitive obesity (738 males, 760 females). The prevalence of insulinsensitive obesity was $18 \%$ (20.5\% in males and $16.1 \%$ in females). Descriptive data on the insulin-resistant/-sensitive general obese phenotypes are presented in Table 1 .

Table 2 shows the odds ratios (ORs) of MVD across 
Table 2 Microvascular risk factors in insulin-sensitive/-resistant general obese phenotypes among middle-aged and older people

\begin{tabular}{|c|c|c|c|c|c|c|c|c|c|c|c|}
\hline Group & \multicolumn{3}{|c|}{ Normal weight } & \multicolumn{4}{|c|}{ Overweight } & \multicolumn{4}{|c|}{ Obese } \\
\hline \multicolumn{12}{|l|}{$45 \leq$ years $<65$} \\
\hline Unadjusted & 1.000 & $\begin{array}{c}2.463 \\
(0.987-6.147)\end{array}$ & 0.053 & $\begin{array}{c}1.495 \\
(0.805-2.774)\end{array}$ & 0.203 & $\begin{array}{c}3.057 \\
(1.467-6.370)\end{array}$ & 0.003 & $\begin{array}{c}2.154 \\
(1.214-3.821)\end{array}$ & 0.009 & $\begin{array}{c}4.329 \\
(2.592-7.228)\end{array}$ & $<0.001$ \\
\hline Model 2 & 1.000 & $\begin{array}{c}1.987 \\
(0.770-5.126)\end{array}$ & 0.156 & $\begin{array}{c}1.225 \\
(0.643-2.334)\end{array}$ & 0.537 & $\begin{array}{c}2.226 \\
(1.022-4.850)\end{array}$ & 0.044 & $\begin{array}{c}1.647 \\
(0.901-3.012)\end{array}$ & 0.105 & $\begin{array}{c}2.594 \\
(1.442-4.666)\end{array}$ & 0.001 \\
\hline \multicolumn{12}{|l|}{$\geq 65$ years } \\
\hline Unadjusted & 1.000 & $\begin{array}{c}1.474 \\
(0.411-5.287)\end{array}$ & 0.552 & $\begin{array}{c}2.030 \\
(0.969-4.252)\end{array}$ & 0.061 & $\begin{array}{c}2.024 \\
(0.758-5.405)\end{array}$ & 0.160 & $\begin{array}{c}2.343 \\
(1.142-4.808)\end{array}$ & 0.020 & $\begin{array}{c}2.775 \\
(1.395-5.520)\end{array}$ & 0.004 \\
\hline
\end{tabular}

Data are presented as OR $(95 \% \mathrm{Cl})$. Model 2: multivariable model adjusted for age, FPG, 2hPPG, SBP, DBP, HDL-C, LDL-C, CHOL, TG, smoking and alcohol. FPG, fasting plasma glucose; 2hPPG, 2-hour postprandial blood glucose; SBP, systolic blood pressure; DBP, diastolic blood pressure; CHOL, total cholesterol; LDL-C, low-density lipoprotein cholesterol; HDL-C, high-density lipoprotein cholesterol; $\mathrm{TG}$, triglyceride; OR, odds ratio; $\mathrm{Cl}$, confidence interval.

obese status in the multivariable adjusted models, and a comparison with insulin-sensitive normal weight individuals. Among middle-aged people (aged 45-65 years), a higher prevalence of retinal microvascular abnormalities was directly associated with both insulin-resistant obesity and overweight. For instance, insulin-resistant overweight participants had an OR of 2.226 [95\% confidence interval (CI): 1.022-4.850, $\mathrm{P}=0.044]$ for retinal microvascular abnormalities, and the relative OR was 2.594 (95\% CI: $1.442-4.666, \mathrm{P}=0.001)$ in insulin-resistant obesity. In older people (aged 65-79 years), obese subjects had a markedly higher risk for retinal microvascular abnormalities in both insulin-sensitive (OR: 2.259, 95\% CI: 1.041-4.904, $\mathrm{P}=0.039)$ and in insulin-resistant (OR: 2.356, 95\% CI: 1.064-5.218, $\mathrm{P}=0.035$ ) individuals. There were insufficient cases of MVD to be calculated in the young group (aged 20-45 years). The risk of MVD in the insulin-sensitive obesity subgroup was similar to that in the insulin-sensitive normal weight group of middle-aged subjects.

In the middle-aged subjects, compared with insulinsensitive normal waist individuals, both insulin-resistant normal waist individuals and insulin-sensitive/-resistant abdominally obese individuals had increased risks for retinal microvascular abnormalities in women (OR: 2.769, 2.061, 3.322; 95\% CI: $1.106-6.933,1.004-4.233,1.645-6.709$; P values: $0.030,0.049,<0.001)$. However, the risk for retinal microvascular abnormalities in men was not increased. In the older group, insulin-resistant abdominal obesity in men was associated with a significantly higher risk (OR: 4.162, 95\% CI: $1.420-12.195, \mathrm{P}=0.009$ ), compared with insulinsensitive normal waist subjects (Table 3), but not in male or female insulin-sensitive abdominally obese individuals.

A subgroup of obese individuals showed better insulin sensitivity than the normal weight individuals, and this phenotype was described as insulin-sensitive obesity. In this subgroup (Table 4), compared with the young subjects (20-45 years), older people (65-79 years) had an elevated risk of MVD (OR: 11.326, 95\% CI: 2.377-53.956, $\mathrm{P}=0.002$ ), but middle-aged subjects showed no differences compared to the young.

Stratification according to smoking status can be an important way to examine the association between obesity and the risk of MVD. Because there were few smokers among the women (61 smokers, $1.3 \%$ ), the subgroup analysis (Table 5) for non-smokers or smokers was only conducted for the men (1,679 smokers, 46.6\%). No significant differences were observed in the first three models in relation to smoking in men. Compared with insulin-sensitive normal weight, insulin-resistant obesity was associated with an elevated risk of MVD in nonsmokers (OR: 3.769, 95\% CI: 1.533-9.269, $\mathrm{P}=0.004$ ), and insulin-sensitive obese subjects showed a rising trend (OR: 
Table 3 Microvascular risk factors in insulin-sensitive/-resistant abdominal obese phenotypes among middle-aged and older people

\begin{tabular}{|c|c|c|c|c|c|c|c|}
\hline Group & \multicolumn{3}{|c|}{ Normal waist } & \multicolumn{4}{|c|}{ Big waist } \\
\hline \multicolumn{8}{|l|}{$45 \leq$ years $<65$} \\
\hline \multicolumn{8}{|l|}{ Men } \\
\hline $\mathrm{N}(\%)$ & $141(1.7)$ & $299(3.6)$ & - & $291(3.5)$ & - & $466(5.6)$ & - \\
\hline Adjusted & 1.000 & $1.460(0.635-3.356)$ & 0.372 & $1.339(0.567-3.166)$ & 0.506 & 1.741 (0.799-3.792) & 0.163 \\
\hline \multicolumn{8}{|l|}{ Women } \\
\hline N (\%) & $83(1.0)$ & $258(3.1)$ & - & $216(2.6)$ & - & $407(4.9)$ & - \\
\hline Unadjusted & 1.000 & $3.101(1.287-7.471)$ & 0.012 & $2.516(1.254-5.048)$ & 0.009 & 4.957 (2.634-9.328) & $<0.001$ \\
\hline \multicolumn{8}{|l|}{ Men } \\
\hline $\mathrm{N}(\%)$ & $432(5.2)$ & $241(2.9)$ & - & 707 (8.5) & - & $1,272(15.3)$ & - \\
\hline Unadjusted & 1.000 & $0.531(0.121-2.333)$ & 0.402 & $1.672(0.599-4.664)$ & 0.326 & $3.249(1.393-7.578)$ & 0.006 \\
\hline Adjusted & 1.000 & $0.617(0.133-2.866)$ & 0.538 & $1.372(0.430-4.381)$ & 0.593 & $4.162(1.420-12.195)$ & 0.009 \\
\hline \multicolumn{8}{|l|}{ Women } \\
\hline N (\%) & $648(7.8)$ & $374(4.5)$ & - & $648(7.8)$ & - & $914(11.0)$ & - \\
\hline Unadjusted & 1.000 & $0.567(0.069-4.662)$ & 0.597 & $1.005(0.426-2.370)$ & 0.992 & $1.477(0.657-3.321)$ & 0.345 \\
\hline
\end{tabular}

Data are presented as OR $(95 \% \mathrm{Cl})$ if not otherwise specified. Model 2: multivariable model adjusted for age, FPG, 2hPPG, SBP, DBP, HDL-C, LDL-C, CHOL, TG, smoking and alcohol. FPG, fasting plasma glucose; 2hPPG, 2-hour postprandial blood glucose; SBP, systolic blood pressure; DBP, diastolic blood pressure; CHOL, total cholesterol; LDL-C, low-density lipoprotein cholesterol; HDL-C, high-density lipoprotein cholesterol; TG, triglyceride; OR, odds ratio; $\mathrm{Cl}$, confidence interval.

Table 4 Microvascular risk factors in insulin-sensitive obese phenotypes

\begin{tabular}{|c|c|c|c|c|c|}
\hline Group & $<45$ years & \multicolumn{2}{|c|}{$45 \leq$ years $<65$} & \multicolumn{2}{|c|}{$\geq 65$ years } \\
\hline $\mathrm{N}(\%)$ & $315(21.0)$ & \multicolumn{2}{|c|}{$1,002(66.9)$} & \multicolumn{2}{|c|}{$181(12.1)$} \\
\hline Unadjusted & 1.000 & $4.169(0.984-17.664)$ & 0.053 & $16.223(3.703-71.072)$ & $<0.001$ \\
\hline Model 3 & 1.000 & $3.349(0.770-14.578)$ & 0.107 & $11.326(2.377-53.956)$ & 0.002 \\
\hline
\end{tabular}

Model 3: multivariable model adjusted for FPG, 2hPPG, SBP, DBP, HDL-C, LDL-C, CHOL, TG, smoking, alcohol, BMI and HOMA-IR. FPG, fasting plasma glucose; 2hPPG, 2-hour postprandial blood glucose; SBP, systolic blood pressure; DBP, diastolic blood pressure; CHOL, total cholesterol; LDL-C, low-density lipoprotein cholesterol; HDL-C, high-density lipoprotein cholesterol; TG, triglyceride; BMI, body mass index; HOMA-IR, HOMA-IR, homeostasis model assessment-estimated insulin resistance; OR, odds ratio; Cl, confidence interval. 
Table 5 Microvascular risk factors in insulin-sensitive/-resistant obese phenotypes according to smoking status in men

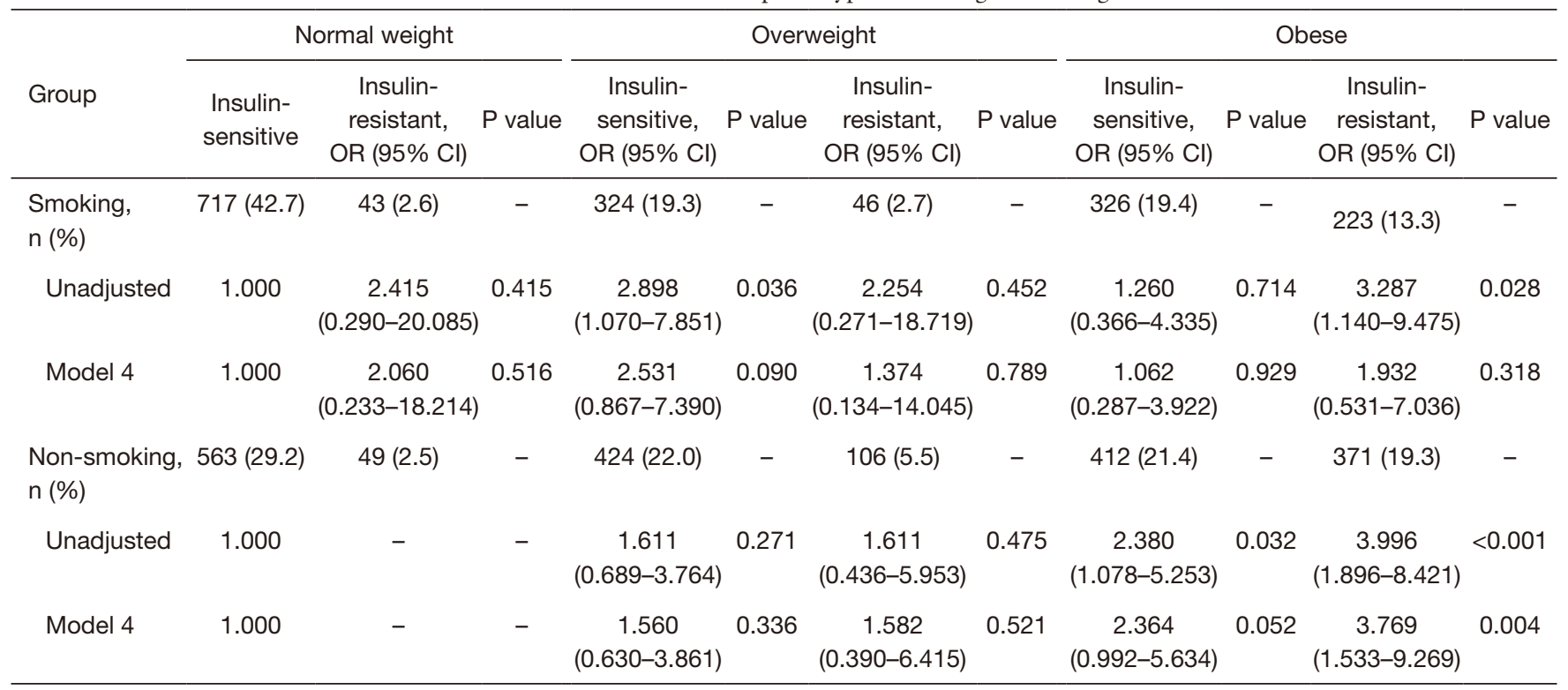

Model 4: multivariable model adjusted for age FPG, 2hPPG, SBP, DBP, HDL-C, LDL-C, CHOL, TG, and alcohol. FPG, fasting plasma glucose; 2hPPG, 2-hour postprandial blood glucose; SBP, systolic blood pressure; DBP, diastolic blood pressure; CHOL, total cholesterol; LDL-C, low-density lipoprotein cholesterol; HDL-C, high-density lipoprotein cholesterol; TG, triglyceride; OR, odds ratio; Cl, confidence interval.

\subsection{4, 95\% CI: 0.992-5.634, $\mathrm{P}=0.052$ ).}

Table 6 presents the raw and adjusted ORs for the likelihood of MVD across insulin-sensitive/-resistant obesity among women classified by menopause status. There was no significant difference observed among postmenopausal women who went into menopause less than 10 years previously. However, in both premenopausal and postmenopausal women whose menopause status was more than 10 years, the insulin-resistant obese subjects were significantly associated with a greater likelihood of MVD even after full adjustment (OR: 16.128, 95\% CI: 1.757-148.042, $\mathrm{P}=0.014$; OR: 2.992, 95\% CI: 1.252-7.148, $\mathrm{P}=0.014)$.

\section{Discussion}

Worldwide, the prevalence of overweight and obesity combined rose by $27.5 \%$ for adults and $47.1 \%$ for children between 1980 and 2013. The number of overweight and obese individuals increased from 857 million in 1980 to 2.1 billion in 2013. Based on an analysis of the China Health and Nutrition Survey data, Xi et al. (14) reported an increase of $67 \%$ in the prevalence of overweight from $9.4 \%$ in 1993 to $15.7 \%$ in 2009 and an increase of $168 \%$ in the prevalence of obesity from $4.0 \%$ in 1993 to $10.7 \%$ in 2009, respectively (15). In 2000, the WHO Western Pacific Region (WHO-WPR) proposed a revised guideline that defined BMI of 23 and $25 \mathrm{~kg} / \mathrm{m}^{2}$ as overweight and obesity, respectively, in Asians (16). Thus, we recruited overweight/ obese individuals based on this guideline.

As far as we know, obesity, especially if accompanied by $\mathrm{IR}$, is associated with a high risk of cardiovascular events. But what about the significant influence of overweight/ obesity on human MVD? In our study, obesity was associated with an elevated prevalence of MVD in the elderly (65-79 years), regardless of the presence or absence of IR. However, in middle-aged people (45-65 years), only women with abdominal obesity had a markedly high risk for MVD, whether or not obesity was accompanied by IR.

Many comorbidities have been positively correlated to overweight/obesity; for example, obesity-related glomerulopathy (ORG), cardiomyopathy and lacunar infarction. Proteinuria is a remarkable clinical manifestation of ORG, as with diabetic nephropathy. Previous study has found that ORG defined morphologically as focal segmental glomerulosclerosis (FSGS) and glomerulomegaly, is characterized by podocyte hypertrophy and reduced 
Table 6 Microvascular risk factors in insulin-sensitive/-resistant obese phenotypes according to menopausal status in women

\begin{tabular}{|c|c|c|c|c|c|c|c|c|c|c|c|}
\hline Group & \multicolumn{3}{|c|}{ Normal weight } & \multicolumn{4}{|c|}{ Overweight } & \multicolumn{4}{|c|}{ Obese } \\
\hline $\begin{array}{l}\text { Pre-menopause, } \\
\text { n (\%) }\end{array}$ & $\begin{array}{l}1,121 \\
(19.6)\end{array}$ & $131(2.3)$ & - & $526(9.2)$ & - & $182(3.2)$ & - & 395 (6.9) & - & $381(6.6)$ & \\
\hline Model 2 & 1.000 & $\begin{array}{c}17.353 \\
(1.432-210.283)\end{array}$ & 0.025 & - & - & $\begin{array}{c}8.149 \\
(0.672-98.876)\end{array}$ & 0.099 & $\begin{array}{c}5.262 \\
(0.504-54.903)\end{array}$ & 0.165 & $\begin{array}{c}16.128 \\
(1.757-148.042)\end{array}$ & 0.014 \\
\hline $\begin{array}{l}\leq 10 \text { years after } \\
\text { menopause, } \mathrm{n} \\
(\%)\end{array}$ & $530(9.2)$ & $79(1.4)$ & - & $323(5.6)$ & - & $113(2.0)$ & - & $295(5.1)$ & - & $324(5.6)$ & \\
\hline Model 2 & 1.000 & $\begin{array}{c}0.382 \\
(0.043-3.378)\end{array}$ & 0.387 & $\begin{array}{c}0.403 \\
(0.107-1.519)\end{array}$ & 0.180 & $\begin{array}{c}1.534 \\
(0.485-4.853)\end{array}$ & 0.467 & $\begin{array}{c}0.814 \\
(0.292-2.268)\end{array}$ & 0.694 & $\begin{array}{c}0.759 \\
(0.272-2.122)\end{array}$ & 0.600 \\
\hline $\begin{array}{l}>10 \text { years after } \\
\text { menopause, } n \\
(\%)\end{array}$ & $391(6.8)$ & $64(1.1)$ & - & $227(4.0)$ & - & 109 (1.9) & - & $237(4.1)$ & - & $314(5.5)$ & \\
\hline Unadjusted & 1.000 & $\begin{array}{c}3.094 \\
(0.996-9.607)\end{array}$ & 0.051 & $\begin{array}{c}1.901 \\
(0.757-4.773)\end{array}$ & 0.171 & $\begin{array}{c}2.397 \\
(0.828-6.936)\end{array}$ & 0.107 & $\begin{array}{c}2.645 \\
(1.121-6.245)\end{array}$ & 0.026 & $\begin{array}{c}3.240 \\
(1.470-7.141)\end{array}$ & 0.004 \\
\hline Model 2 & 1.000 & $\begin{array}{c}2.832 \\
(0.848-9.456)\end{array}$ & 0.091 & $\begin{array}{c}2.154 \\
(0.827-5.613)\end{array}$ & 0.116 & $\begin{array}{c}2.653 \\
(0.844-8.335)\end{array}$ & 0.116 & $\begin{array}{c}2.627 \\
(1.051-6.565)\end{array}$ & 0.039 & $\begin{array}{c}2.992 \\
(1.252-7.148)\end{array}$ & 0.014 \\
\hline
\end{tabular}

Model 2: multivariable model adjusted for age, FPG, 2hPPG, SBP, DBP, HDL-C, LDL-C, CHOL, TG, smoking, and alcohol. FPG, fasting plasma glucose; 2hPPG, 2-hour postprandial blood glucose; SBP, systolic blood pressure; DBP, diastolic blood pressure; CHOL, total cholesterol; LDL-C, low-density lipoprotein cholesterol; HDL-C, high-density lipoprotein cholesterol; TG, triglyceride; OR, odds ratio; Cl, confidence interval.

podocyte density, increased mesangial matrix and mesangial cell proliferation (17). Cardiac failure often accompanies obesity cardiomyopathy, independent of hypertension, coronary artery disease, dyslipidemia, and hyperglycemia. Previous study has shown that obesity cardiomyopathy (adipositas cordis) occurs because of an accumulation of adipose tissue between myofibers and degradation of myocytes. The degeneration of the myocardium could be due to lipotoxicity of free fatty acids in adipose tissue (18). Moreover, a new finding is that obesity is an independent risk factor for the development of cerebral microangiopathy (19). Obesityrelated lacunar infarction, which may lead to depression, cognitive dysfunction, has become a public concern in recent years. It has been proposed that hormones (i.e., leptin, adiponectin, resistin) and cytokines (tumor necrosis factor-alpha, interleukin-6) produced by adipose tissue are the link between obesity and obesity-related lacunar infarction (20). Although there is some research on the potential mechanisms of ORG, obesity cardiomyopathy, and lacunar infarction, separating the disorders and the specific roles each play in disease progression is currently very difficult. The overall pathophysiology of obesity is multifactorial. Addressing this, we hypothesized that obesity-related diseases may not be only associated with hyperglycemia, hypertension, and hyperlipidemia. Obesityrelated microangiopathy may be a significant mechanism in obesity-related organ damage. Obesity maybe analogous to diabetes mellitus, which also has chronic microvascular complications. According to our research, obesity, independent of IR and especially in older people and middle-aged women with abdominal obesity, is closely related to a higher risk of MVD. Weight control should 
be a recommendation for preventing the microvascular complications of obesity.

As we noted above, insulin sensitivity is the first apparent risk factor and is a major risk factor. Insulin sensitivity varies in obesity across the age groups. In the present study, older obese individuals who were insulin-sensitive had a higher risk for microangiopathy independent of BMI and HOMAIR status, whereas middle-aged individuals were not at higher risk compared with young people. Therefore, agespecific insulin-sensitive subjects have different metabolic characteristics, especially in regard to microvascular abnormalities. Therefore, age is probably the second risk factor for obesity-related microangiopathy.

The present study also showed that abdominal obesity was most associated with increased risk for MVD in women, especially middle-aged subjects, but not in men. Our study also revealed that premenopausal women with IR, regardless of weight, were significantly associated with a greater likelihood of MVD. Moreover, we found that obese postmenopausal women whose menopause status was more than 10 years, regardless of insulin sensitivity or resistance, were significantly associated with a greater likelihood of MVD. Estrogen has a role in transcending female sexual development and reproduction, linking the pathogenesis of obesity-related MVD to female sex.

In addition, smoking was a concern in the analyses of body weight and death, because it was associated with decreased body weight but an increased risk of death (21). Statistical adjustment for smoking status was often insufficient. An additional concern was reverse causation, whereby underlying chronic disease or frailty both causes weight loss and elevates the risk of death. Thus, to demonstrate the obesity paradox, the present study was stratified according to smoking status in men. Because few women were smokers $(61,1.3 \%)$, we did not conduct the subgroup analysis in women. The result suggested that nonsmoking men tended to present a higher risk of MVD. An association between obesity and MVD was not observed in smokers. Smoking may not be a possible risk factor for MVD.

\section{Strength and limitations}

The major strength of the present study is the large, wellcharacterized population of 8,313 participants, which was fairly representative of most adults living in southeastern China. Unlike previous studies of obesity and macrovascular diseases, our study focused on the relationship between obesity and MVD. Because of the important effects of insulin sensitivity, we divided the overweight/obese subjects into insulin-sensitive and insulin-resistant groups. However, limitations should still be considered. One major limitation is that due to the cross-sectional nature of the study, no causal inference can be drawn. Prospective studies are needed to clarify the precise interrelationship. Secondly, the number of cases of MVD among young people in this study was few, which limits its generalizability to similar age groups of other ethnicities.

\section{Conclusions}

According to our research, obesity, regardless of the presence or absence of IR, and especially for older people and abdominally obese women, is closely related to a significantly higher risk of MVD. Obesity may be similar to diabetes mellitus, which also has chronic complications, causing not only cardiovascular diseases but also MVD. Direct ophthalmoscopy may be a noninvasive and meaningful method of early screening for obesity-related MVD. Insulin-sensitive obesity, like IGT, is also a preexisting state of abnormal metabolic obesity. Meanwhile, age, insulin sensitivity, waist circumference, and estrogen could be conceivable risk factors for the association of obesity with MVD to a certain extent, but not smoking. Among these factors, weight loss is the sole controllable risk, which can further improve insulin sensitivity and waist circumference. We suggest that weight loss is an important approach to preventing obesity-related MVD, especially in middle-aged abdominally obese women and obese older people.

\section{Acknowledgments}

Funding: This study was supported by grants from the National Key Research and Development Program of China (2018YFC2001100-5), Chinese Medical Association Foundation and Chinese Endocrine Society (No. 12020240314), National Natural Science Foundation of China (No. 81270874), Natural Science Foundation of Fujian Province (No. 2011J06012, 2012J01322), and the Provincial Health and Family Planning Commission of Fujian Province (No. 2013-ZQN-ZD-3). The funders had no role in study design, data collection and analysis, decision to publish, or preparation of the manuscript. 


\section{Footnote}

Reporting Checklist: The authors have completed the STROBE reporting checklist. Available at http://dx.doi. org/10.21037/apm-20-447

Data Sharing Statement: Available at http://dx.doi. org/10.21037/apm-20-447

Conflicts of Interest: All authors have completed the ICMJE uniform disclosure form (available at http://dx.doi. org/10.21037/apm-20-447). The authors have no conflicts of interest to declare.

Ethical Statement: The authors are accountable for all aspects of the work in ensuring that questions related to the accuracy or integrity of any part of the work are appropriately investigated and resolved. Written informed consent was given by every participant and the study obtained ethics approval from the Ethics Committee of Fujian Provincial Hospital. The number/ID of the approval is (K2009-12-020). The study was conducted in accordance with the Declaration of Helsinki (as revised in 2013).

Open Access Statement: This is an Open Access article distributed in accordance with the Creative Commons Attribution-NonCommercial-NoDerivs 4.0 International License (CC BY-NC-ND 4.0), which permits the noncommercial replication and distribution of the article with the strict proviso that no changes or edits are made and the original work is properly cited (including links to both the formal publication through the relevant DOI and the license). See: https://creativecommons.org/licenses/by-nc-nd/4.0/.

\section{References}

1. Stevens GA, Singh GM, Lu Y, et al. National, regional, and global trends in adult overweight and obesity prevalences. Popul Health Metr 2012;10:22.

2. Popkin BM, Adair LS, Ng SW. Global nutrition transition and the pandemic of obesity in developing countries. Nutr Rev 2012;70:3-21.

3. Popkin BM, Slining MM. New dynamics in global obesity facing low- and middle-income countries. Obes Rev 2013;14:11-20.

4. Vona-Davis L, Howard-McNatt M, Rose DP. Adiposity, type 2 diabetes and the metabolic syndrome in breast cancer. Obes Rev 2007;8:395-408.

5. Stefan N. Identification and Characterization of
Metabolically Benign Obesity in Humans. Arch Intern Med 2008;168:1609.

6. Meigs JB, Wilson PWF, Fox CS, et al. Body Mass Index, Metabolic Syndrome, and Risk of Type 2 Diabetes or Cardiovascular Disease. J Clin Endocrinol Metab 2006;91:2906-12.

7. Calori G, Lattuada G, Piemonti L, et al. Prevalence, Metabolic Features, and Prognosis of Metabolically Healthy Obese Italian Individuals: The Cremona Study. Diabetes Care 2011;34:210-5.

8. Arnlöv J, Ingelsson E, Sundström J, et al. Impact of body mass index and the metabolic syndrome on the risk of cardiovascular disease and death in middle-aged men. Circulation 2010;121:230-6.

9. Khan UI, Wang D, Thurston RC, et al. Burden of subclinical cardiovascular disease in "metabolically benign" and "at-risk" overweight and obese women: The Study of Women's Health Across the Nation (SWAN). Atherosclerosis 2011;217:179-86.

10. Arnlöv J, Sundstrom J, Ingelsson E, et al. Impact of BMI and the Metabolic Syndrome on the Risk of Diabetes in Middle-Aged Men. Diabetes Care 2011;34:61-5.

11. Taylor B, Rochtchina E, Wang JJ, et al. Body mass index and its effects on retinal vessel diameter in 6-year-old children. Int J Obes (Lond) 2007;31:1527-33.

12. Wong TY, Shankar A, Klein R, et al. Prospective cohort study of retinal vessel diameters and risk of hypertension. BMJ 2004;329:79.

13. Wong TY, Duncan BB, Golden SH, et al. Associations between the metabolic syndrome and retinal microvascular signs: the Atherosclerosis Risk In Communities study. Invest Ophthalmol Vis Sci 2004;45:2949-54.

14. Xi B, Liang Y, He T, et al. Secular trends in the prevalence of general and abdominal obesity among Chinese adults, 1993-2009. Obes Rev 2012;13:287-96.

15. James PT, Leach R, Kalamara E, et al. The Worldwide Obesity Epidemic. Obes Res 2001;9:228S-233S.

16. Ko GTC, Tang JSF. Waist Circumference and BMI Cut-off Based on 10-year Cardiovascular Risk: Evidence for "Central Pre-Obesity"**. Obesity 2007;15:2832-9.

17. D'Agati VD, Chagnac A, de Vries APJ, et al. Obesityrelated glomerulopathy: clinical and pathologic characteristics and pathogenesis. Nat Rev Nephrol 2016;12:453-71.

18. Ussher JR. The role of cardiac lipotoxicity in the pathogenesis of diabetic cardiomyopathy. Expert Rev 
Cardiovasc Ther 2014;12:345-58.

19. Okroglic S, Widmann CN, Urbach H, et al. Clinical Symptoms and Risk Factors in Cerebral Microangiopathy Patients. Minnerup J, editor. PLoS One 2013;8:e53455.

20. Yamashiro K, Tanaka R, Tanaka Y, et al. Visceral fat

Cite this article as: Lin W, Rao H, Huang H, Yao J, Liang J, Li L, Wen J, Chen G. Relationship between insulin-sensitive obesity and retinal microvascular abnormalities. Ann Palliat Med 2021;10(2):1031-1041. doi: 10.21037/apm-20-447 accumulation is associated with cerebral small vessel disease. Eur J Neurol 2014;21:667-73.

21. Tobias DK, Pan A, Jackson CL, et al. Body-Mass Index and Mortality among Adults with Incident Type 2 Diabetes. N Engl J Med 2014;370:233-44. 